Pacific Journal of Mathematics

FINDING A MAXIMAL SUBALGEBRA ON WHICH THE TWO 


\title{
FINDING A MAXIMAL SUBALGEBRA ON WHICH THE TWO ARENS PRODUCTS AGREE
}

\author{
JULIEN O. HENNEFELD
}

\begin{abstract}
Arens has given two ways of defining a Banach algebra product on the second dual of a Banach algebra $\mathscr{A}$. In this paper we give a construction for finding a maximal subalgebra on which the two Arens products agree. Moreover, we give an example which shows that there is not necessarily a unique maximal subalgebra on which the two Arens products agree. This example is a Banach algebra whose second dual has a non unique element $I$ which is simultaneously a right identity under the first Arens product and a left identity under the second Arens product.
\end{abstract}

1. Preliminaries. The two Arens products are defined according to the following rules. Let $\mathscr{A}$ be a Banach algebra. Let $A, B \in \mathscr{A}$, $f \in \mathscr{A}^{*}, F, G \in \mathscr{A}^{* *}$.

DeFinITION 1.1.

$\left(f *_{1} A\right) B=f(A B)$ This defines $f *_{1} A$ as an element of $\mathscr{A}^{*}$. $\left(G *_{1} f\right) A=G\left(f *_{1} A\right)$ This defines $G *_{1} f$ as an element of $\mathscr{A}^{*}$. $\left(F *_{1} G\right) f=F\left(G *_{1} f\right)$ This defines $F *_{1} G$ as an element of $\mathscr{A}^{* *}$. We will call $F *_{1} G$ the first or the $m_{1}$ product.

Definition 1.2. $\left(f *_{2} A\right) B=f(B A) ;\left(F *_{2} f\right) A=F\left(f *_{2} A\right) ;\left(F *_{2} G\right) f=$ $G\left(F *_{2} f\right)$.

We will call $F *_{2} G$ the second or the $m_{2}$ product.

Proposition 1.3. If $\mathscr{A}$ has an approximate identity, then $\mathscr{A}^{* *}$ has an element $I$ which is simultaneously a right $m_{1}$ identity and a left $m_{2}$ identity. Call such an element $I$ a simultaneous identity.

Proof. $\mathscr{A}^{* *}$ has a right $m_{1}$ identity by $[2$, p. 146] the proof that it also has a left $m_{2}$ identity is similar.

EXAMPLE 1.4. A simultaneous right $m_{1}$ and left $m_{2}$ identity, unlike a two-sided identity, is not necessarily unique.

Let $X=c_{0} \bigoplus_{\text {sup }} \ell^{1}$. Let $\left\{x_{1}, x_{2}, x_{3}, x_{4}, \cdots\right\}$ be the basis $\left\{d_{1}, e_{1}, d_{2}, e_{2}, \cdots\right\}$ where $\left\{d_{i}\right\}$ and $\left\{e_{i}\right\}$ are the canonical bases for $c_{0}$ and $\ell^{1}$ respectively. Let $\mathscr{D}$ be the norm closure of operators in $\mathscr{B}(X)$ which have a finite matrix with respect to $\left\{x_{i}\right)$. For each $f \in \mathscr{D}^{*}$ we can associate a matrix $\left(f_{i j}\right)$ by defining $f_{i j}=f\left(E_{i j}\right)$ when 
$E_{i j}$ is the matrix in $\mathscr{D}$ with a 1 in the $i j^{\text {th }}$ place and 0 's elsewhere. $\mathscr{D}$ has an approximate indentity, namely the operators $E_{n}$ with 1's down the first $n$ entries on the diagonal and 0's elsewhere.

Let $T_{n}$ be the matrix with 1 's in the $j+1, j^{\text {th }}$ slots for $j=$ $1,3,5, \cdots, 2 n-1$ and 0 's elsewhere. Clearly $\|T\|=n$ and so by the Hahn Banach theorem there exists an $f_{n} \in \mathscr{D}^{*}$ of norm one with $f_{n}\left(T_{n}\right)=n$. Since $f_{n}$ has norm one, each of its entries must have modulus $\leqq 1$. This can be seen directly or from [7, Prop. 2.6]. Hence the matrix for $f_{n}$ must have $j+1, j^{\text {th }}$ entries $=1$ for $j=$ $1,3, \cdots, 2 n-1$.

By the weak star compactness of the unit ball of $\mathscr{D}^{*}$ there exists an $f$ which is a weak star cluster point of the $f_{n}$. Note that the $j+1, j^{\text {th }}$ entries of $f$ must all be 1 , because if $f_{m+1, m} \neq 1$ for some $m$, then the weak star neighborhood of $f$ given by $\mathscr{N}\left(f ; E_{m+1, m} ; \varepsilon\right)$ would not contain infinitely many $f_{n}$ for $\varepsilon$ small. It is clear that $f$ is not in the subspace of $\mathscr{D}^{*}$ spanned by those functionals whose matrices have either a finite number of rows or columns. Hence, there exists and $H \in \mathscr{D}^{* *}$ such that $H(f)=1$ and $H(g)=0$ if the matrix for $g$ has a finite number of rows or a finite number of columns.

Note that $I *_{1} H=0$ because for arbitrary $g \in \mathscr{D}^{*},\left(I *_{1} H\right) g=$ $\lim _{n}\left(E_{n} *_{1} H\right) g$ by the left weak star continuity of $m_{1}$. See [1]. This equals $\lim _{n} E_{n}\left(H *_{1} g\right)=\lim _{n}\left(H *_{1} g\right) E_{n}=\lim _{n} H\left(g *_{1} E_{n}\right)$. But it is easily seen that for each $n$, the matrix for the functional $g *_{1} E_{n}$ has the same first $n$ rows as that of $g$ and zeroes elsewhere. This can be computed directly. Hence $H\left(g *_{1} E_{n}\right)=0$ and so $I *_{1} H=0$. Similarly it can be seen that $\left(H *_{2} I\right) g=\lim \left(H *_{2} E_{n}\right) g=\lim E_{n}\left(H *_{2} g\right)=\lim \left(H *_{2} g\right) E_{n}=$ $\lim H\left(g *_{2} E_{n}\right)$ and that the functional $g *_{2} E_{n}$ has as its matrix the first $n$ columns of $g$ and zeroes elsewhere. Thus $H *_{2} I=0$.

From the fact that $I *_{1} H=0$ it follows that $G *_{1} H=0$ for all $G \in \mathscr{D}^{* *}$ since $G *_{1} H=\left(G *_{1} I\right) *_{1} H=G *_{1}\left(I *_{1} H\right)$. Similarly, $H *_{2} I=0$ implies $H *_{2} G=0$ for all $G \in \mathscr{D}^{* *}$. It is easy to see that $H+I$ is a simultaneous right $m_{1}$ and left $m_{2}$ identity.

2. The main result. Let $\mathscr{A}$ be a Banach algebra and suppose the two Arens products agree on $\mathscr{B}$ where $\mathscr{A} \subset \mathscr{B} \subset \mathscr{A}^{* *}$. Then by Zorn's lemma, it follows that there exists an algebra $\mathscr{C}$ with $\mathscr{B} \subset \mathscr{M} \subset \mathscr{A}^{* *}$ such that the two Arens products agree on $\mathscr{M}$ and $\mathscr{C}$ is maximal with respect to this property.

ExAmple 2.1. Let $\mathscr{D}$ be the same Banach algebra as in Example 1.4. Then there is not a unique maximal subalgebra of $\mathscr{D}^{* *}$ on which the Arens products agree. Note that the Arens products agree on the algebra generated by $[\mathscr{D}, I]$. Also they agree on the 
algebra generated by $[\mathscr{D}, H]$, since they agree if one factor is in $\mathscr{D}$, and also $H *_{1} H=H *_{2} H=0$. However the Arens products cannot agree on any algebra containing $I$ and $H$, since $I *_{1}(I+H)=I$ but $I *_{2}(I+H)=I+H$.

Definition 2.2. Let $\mathscr{A}$ be a Banach algebra and $E_{\alpha}$ an approximate identity with weak star limit $I$ in $\mathscr{A}^{* *}$. Then $E_{\alpha}$ is called projecting if for each $F \in \mathscr{A}^{* *}, E_{\alpha}{ }_{1} F *_{1} E_{\beta}$ is in $\mathscr{A}$ for $E_{\alpha}$ and $E_{\beta}$ sufficiently far out.

THEOREM 2.3. Let $E_{\alpha}$ be a projecting weak identity for $\mathscr{A}$ and let $I *_{1}\left(F *_{2} I\right)=F *_{2} I$ for all $F \in A^{* *}$. Then

(1) $m_{1}=m_{2}$ on $\mathscr{N}$ where $\mathscr{N}=\left\{F *_{2} I: F \in \mathscr{A}^{* *}\right\}$

(2) $\mathscr{N}$ is an algebra which is maximal with respect to the property that $m_{1}=m_{2}$.

Proof. One of the difficulties is the fact that mixed Arens products like $\left(F *_{1} G\right) *_{2} H$ are not necessarily associative. In this proof all limits will be in the weak star topology on $\mathscr{A}^{* *}$. We will make frequent use of the fact that the two Arens products agree if one of the factors is in $\mathscr{A}$. Also we will make very careful use of the left weak star continuity of $m_{1}$ and the right weak star continuity of $m_{2}$. Furthermore note that by the hypothesis on $I$, it follows that $I *_{1} V=V$ for any $V \in \mathscr{N}$.

Given $S=F *_{2} I$ and $T=G *_{2} I$ we must show that $S *_{2} T=S *_{1} T$. Note that $S *_{2} T=I *_{1}\left(S *_{2} T\right)$ since $S *_{2} T$ is in $\mathscr{N}$ and equals

Note also that

$$
\left(\lim _{\alpha} E_{\alpha}\right) *_{1}\left(S *_{2} T\right)=\lim _{\alpha}\left[E_{\alpha} *_{1}\left(S *_{2} T\right)\right]
$$

$$
S *_{1} T=\left(I *_{1} S\right) *_{1} T=\lim \left(E_{a} *_{1} S\right) *_{1} T=\lim \left[E_{\alpha} *_{1}\left(S *_{1} T\right)\right] .
$$

Hence it is sufficient to show that $E_{\beta} *_{1}\left(S *_{2} T\right)=E_{\beta} *_{1}\left(S *_{1} T\right)$ for all $E_{\beta}$ far enough out.

But since $E_{\beta} \in \mathscr{A}, E_{\beta} *_{1}\left(S *_{2} T\right)=E_{\beta} *_{2}\left(S *_{2} T\right)$

$=\left(E_{\beta} *_{2} S\right) *_{2} T=\left(E_{\beta} *_{2} S\right) *_{2}\left(I *_{1} T\right)$

$=\left(E_{\beta} *_{2} S\right) *_{2} \lim _{\alpha}\left(E_{\alpha} *_{2} T\right)$ by the left weak star continuity of $m_{1}$

$=\lim _{\alpha}\left[\left(E_{\beta} *_{2} S\right) *_{2}\left(E_{\alpha} *_{2} T\right)\right]$ by the right weak star continuity of $m_{2}$

$=\lim _{\alpha}\left[\left(\left(E_{\beta} *_{2} S\right) *_{2} E_{\alpha}\right) *_{2} T\right]$

$=\lim _{\alpha}\left[\left(\left(E_{\beta} *_{2} S\right) *_{2} E_{\alpha}\right) *_{1} T\right]$ since $E_{\beta} * S * E_{\alpha}$ is in for $E_{\beta}$ and $E_{\alpha}$ far enough out

$=\lim _{\alpha}\left[\left(E_{\beta} *_{2} S\right) *_{2} E_{\alpha}\right] *_{1} T=\left[\left(E_{\beta} *_{2} S\right) *_{2} I\right] *_{1} T$ by weak star continuity

$=\left(E_{\beta} *_{2}\left(S *_{2} I\right)\right) *_{1} T=\left(E_{\beta} *_{2} S\right) *_{1} T$ since $S \in \mathscr{N}$

$=\left(E_{\beta} *_{1} S\right) *_{1} T=E_{\beta} *_{1}\left(S *_{1} T\right)$

and this concludes the proof of part (1). 
For part (2) $\mathscr{N}$ is an algebra because $\left(F *_{2} I\right) *_{2}\left(G *_{2} I\right)=\left(F *_{2} G\right) *_{2} I$ by the associativity of $m_{2}$, and is thus in $\mathscr{N}$. Next suppose that $F \notin \mathscr{N}$. Then $F *_{2} I \neq F$ and yet $F *_{1} I=F$ and so $\mathscr{N}$ is maximal.

3. Applications. For an infinite, Abelian group it is well known [3] that the Arens products never agree on all of $L(G)^{* *}$.

Corollary 3.1. If $G$ is a compact Abelian group, then $L(G)$ satisfies the hypotheses of the above theorem.

Proof. Let $E_{\alpha}$ be an approximate identity for $L(G)$ with weak star limit $I$. By [3, Thm. 2.4] $L(G)$ is a two-sided ideal in $L(G)^{* *}$. So in particular $E_{\alpha}$ will be projecting. It is easily observed that if a Banach algebra $\mathscr{A}$ is commutative, then $F *_{2} A=A *_{2} F$ for all $A \in \mathscr{A}$ and $F \in \mathscr{A}^{* *}$. Then

$$
\begin{aligned}
I *_{1}\left(F *_{2} I\right) & =\lim _{\alpha} \lim _{\beta}\left[E_{\alpha *_{2}}\left(F *_{2} E_{\beta}\right)\right] \\
& =\lim _{\alpha} \lim _{\beta}\left[\left(E_{\alpha} *_{2} F\right) *_{2} E_{\beta}\right]=\lim _{\alpha} \lim _{\beta}\left[\left(F *_{2} E_{\alpha}\right) *_{2} E_{\beta}\right] \\
& =\lim _{\alpha} \lim _{\beta}\left[F *_{2}\left(E_{\alpha} *_{2} E_{\beta}\right)\right]=\lim _{\alpha}\left[F *_{2}\left(E_{\alpha} *_{2} I\right)\right] \\
& =\lim _{\alpha}\left[F *_{2} E_{\alpha}\right]=F *_{2} I .
\end{aligned}
$$

Definition. A shrinking basis $\left\{e_{j}\right\}$ for a Banach space is called boundedly growing if there exists an $\varepsilon>0$ and a positive integer $n$ such that $\left\|x_{1}+\cdots x_{n}\right\|<n-\varepsilon$ whenever the $x_{i}$ 's have norm 1 and are distinct block basic vectors.

CoRollary 3.2. If $X$ has an unconditionally monotone, boundedly growing bases $\left\{e_{j}\right\}$ then $\mathscr{C}$ the algebra of compact linear operators satisfies the hypotheses of the theorem, and $\mathscr{N}$ will consist of those $F \in \mathscr{C}^{* *}$ for which each of the "rows" of $F$ are elements of $X^{*}$ (as opposed to $X^{* *}$ ).

Proof. The operators $E_{n}$, with ones down the first $n$ slots of the diagonal and zeroes elsewhere, form an approximate identity for $\mathscr{C}$. For any $F \in \mathscr{C}^{* *}$ and integers $n, m$ we claim that $E_{n} *_{1} F *_{1} E_{m}$ is in $\mathscr{C}$. To see this first note that for $f \in \mathscr{C}^{*}\left(E_{n} *_{1} F *_{1} E_{m}\right) f=$ $E_{n}\left[\left(F *_{1} E_{m}\right) *_{1} f\right]=\left[\left(F *_{1} E_{m}\right) *_{1} f\right] E_{n}=\left(F *_{1} E_{m}\right)\left(f *_{1} E_{n}\right)=F\left[E_{m} *_{1}\left(f *_{1} E_{n}\right)\right]$. But $E_{m} *_{1}\left(f *_{1} E_{n}\right)$ which is an element of $\mathscr{C}^{*}$ has as its matrix, the matrix obtained from $f$ by replacing by zeroes all rows after the $n^{\text {th }}$ row and all columns after the $m^{\text {th }}$ column. This can be observed directly. Thus $\left(E_{n} * F * E_{m}\right) f=\check{C}(f)$ where $C$ is the compact operator with matrix $\left(C_{i j}\right)$ where $C_{i j}=F\left(g_{i j}\right)$ and $g_{i j}$ has matrix with a one in the $i j^{\text {th }}$ place and zeroes elsewhere. Hence $E_{n} * F * E_{m}=C$. 
From the proof of [7, Prop. 3.3 and Cor. 4.2] it follows that if $X$ has an unconditionally monotone, boundedly growing basis then the matrices with a finite number of rows are dense in $\mathscr{C}^{*}$. See the correction at the end of this paper for details. Thus $I *_{1} F=F$ for any $F \in \mathscr{C}^{* *}$ since $\left(I *_{1} F\right) f=\lim \left(E_{n} *_{1} F\right) f=\lim F\left(f *_{1} E_{n}\right)$ and the matrix for $f *_{1} E_{n}$ can be obtained from that of $f$ by replacing with zeroes all rows after then $n^{\text {th }}$.

To identify $\mathscr{N}$, first note that each $F \in \mathscr{C}^{* *}$ can be regarded as having "rows" which are elements of $X^{* * *}$ and "columns" which are elements of $X^{* *}$. The $n^{\text {th }}$ "row" of $F$ is the restriction of $F$ to the elements of $\mathscr{C}^{*}$ whose matrices have zeros outside the $n^{\text {th }}$ row; "columns" are similarly defined. (Of course, a "row" of $F$ in this sense does not have a sequence of numbers associated with it.)

Then note that $\left(F *_{2} I\right) f=\lim F\left(f *_{2} E_{n}\right)$ and recall that $f *_{2} E_{n}$ has as its matrix the first $n$ columns of $f$. Recall also that the hypotheses imply that the matrices with a finite number of rows are norm dense in $\mathscr{C}^{*}$. Thus $\lim F\left(f *_{2} E_{n}\right)=F(f)$ for all $f$ in $\mathscr{C}^{*}$ if and only if each row of $F$ is in $X^{*}$, since by hypothesis the basis for $X$ is shrinking.

EXAMPLE 3.3. For $X=c_{0}$ or $X=c_{0} \oplus \ell^{p}$ with $1<p<\infty$ the natural basis is boundedly growing. Moreover, $\mathscr{N}$ is strictly contained between $\mathscr{B}(X)$ and $\mathscr{C}^{* *}$, because it will have some elements (with "columns" in $X^{* *}$ ) which won't be in $\mathscr{B}(X)$.

Correction. In [7, Props. 3.2 and 3.3] the assumption that $X$ is reflexive was mistakenly omitted. Of course, the main Theorem 3.2 is not affected, since there $X$ was uniformly convex. Also, in the proof of [7, Cor. 4.2] it was stated that: If $X$ has a boundedly growing, unconditionally monotone basis then the matrices with a finite number of rows are dense in $\mathscr{C}^{*}$. Here is a proof of that fact: Suppose the matrices with a finite number of rows are not dense in $\mathscr{C}^{*}$. We will show that this implies that the basis is not boundedly growing.

First note that there exists and $f \in \mathscr{C}^{*}$ such that $f^{N}$ does not approach 0 , where $f^{N}$ is the matrix formed from $f$ by deleting the first $N$ rows and columns. To see this observe that for $g \in \mathscr{C}^{*}$, if $g^{N} \rightarrow 0$ then $g-g^{N}$ approaches $g$. Thus for $\lambda>0, \exists K:\left\|g-\left(g-g^{K}\right)\right\|<$ $\lambda / 2$. Then since each column of $g$ can be regarded as an element of $X^{*}$ and the basis for $X$ is shrinking, there exists an $M$ such that the matrix consisting of the first $M$ rows of $g-g^{K}$ will be within $\lambda / 2$ of $g-g^{K}$. Therefore, since the matrices with a finite number of rows are assumed to be non dense in $\mathscr{C}^{*}$, there must exist an $f$ for which $f^{N}$ does not approach 0 . Without loss of generality [7, 
Prop. 2.6] we can assume that $\left\|f^{N}\right\| \downarrow 1$.

Given $\varepsilon$ and $n$, let $\delta>0$. Pick $N_{1}:\left\|f^{N_{1}}\right\|<1+\delta$. Since the basis is shrinking, the finite operators are dense in $\mathscr{C}$. Thus there exists an integer $N_{1}^{\prime}>N_{1}$ and a finite operator $T_{1}$ of norm 1 such that $T_{1}$ is concentrated on the manifold $X_{1}=\left[e_{N_{1}}, \cdots, e_{N_{1}^{\prime}}\right]$ and $f^{N_{1}}\left(T_{1}\right)>1$. Let $N_{2}=N_{1}^{\prime}+1$. There exists an operator $T_{2}$ of norm 1 , concentrated on the manifold $X_{2}=\left[e_{N_{2}}, \cdots, e_{N_{2}^{\prime}}\right]$ such that $f^{N_{2}}\left(T_{2}\right)>1$. Repeating this process $n$ times, we can find $T_{1}, \cdots, T_{n}$ such that $f^{N_{k}}\left(T_{k}\right)>1$, and the $T_{k}$ are concentrated on disjoint basic blocks. Hence

$$
\begin{aligned}
n & <f^{N_{1}}\left(T_{1}\right)+\cdots+f^{N_{n}}\left(T_{n}\right)=f^{N_{1}}\left(\mathrm{~T}_{1}+\cdots+T_{n}\right) \\
& \leqq\left\|f^{N_{1}}\right\|\left\|T_{1}+\cdots+T_{n}\right\|
\end{aligned}
$$

thus $n /(1+\delta)<\left\|T_{1}+\cdots+T_{n}\right\|$ and there exists an $x$ of norm 1 , wsere $x=x_{1}+\cdots+x_{n}$ and each $x_{i}$ in $X_{i}$ such that $n /(1+\delta)<$ $\left\|\left(T_{1}+\cdots+T_{n}\right) x\right\|=\left\|T_{1} x_{1}+\cdots+T_{n} x_{n}\right\|$. However, $\delta<0$ was arbitrary. By picking $\delta$ small enough we can assure that $\left\|T_{1} x_{1}+\cdots+T_{n} x_{n}\right\|$ is as close to $n$ as we wish. By unconditional monotonicity, each $\left\|x_{i}\right\| \leqq 1$. Thus each $\left\|T_{i} x_{i}\right\| \leqq 1$ and since the $T_{i} x_{i}$ are from disjoint blocks the basis won't be boundedly growing.

\section{REFERENCES}

1. R. F. Arens, The adjoint of a bilinear operation, Proc. Amer. Math. Soc., 2 (1951), 839-848.

2. F. F. Bongall, J. Duncan, Complete Normed Algebras, Springer-Verlag, N. Y., 1973.

3. P. Civin, Ideals in the second conjugate of a group algebra, Math. Scand., 11 (1962), 161-174.

4. P. Civin and B. Yood, The second conjugate of a Banach algebra as an algebra, Pacific J. Math., 11 (1961), 847-870.

5. S. L. Gulick, Commutativity and ideals in the biduals of topological algebras, Pacific J. Math., 16 (1966).

6. J. Hennefeld, A note on the Arens products, Pacific J. Math., 26 (1968), 115-119.

7. - The Arens products and an imbedding theorem, Pacific J. Math., 29 (1969), $551-563$.

Received September 10, 1974

BOSTON COLLEGE 


\section{PACIFIC JOURNAL OF MATHEMATICS}

\section{EDITORS}

RICHARD ARENS (Managing Editor) University of California

Los Angeles, California 90024

\section{R. A. Beaumont}

University of Washington Seattle, Washington 98105
J. DugundJI Department of Mathematics University of Southern Californıa Los Angeles, California 90007

D. Gilbarg aNd J. Milgram Stanford University Stanford, California 94305

\section{ASSOCIATE EDITORS}

E. F. BeCKENBACH

B. H. NeUmanN

F. Wolf

K. YoSHIDA

\section{SUPPORTING INSTITUTIONS}

UNIVERSITY OF BRITISH COLUMBIA CALIFORNIA INSTITUTE OF TECHNOLOGY UNIVERSITY OF CALIFORNIA MONTANA STATE UNIVERSITY UNIVERSITY OF NEVADA NEW MEXICO STATE UNIVERSITY OREGON STATE UNIVERSITY UNIVERSITY OF OREGON OSAKA UNIVERSITY
UNIVERSITY OF SOUTHERN CALIFORNIA STANFORD UNIVERSITY UNIVERSITY OF TOKYO UNIVERSITY OF UTAH WASHINGTON STATE UNIVERSITY UNIVERSITY OF WASHINGTON AMERICAN MATHEMATICAL SOCIETY NAVAL WEAPONS CENTER 


\section{Pacific Journal of Mathematics \\ Vol. 59, No. 1 \\ May, 1975}

Shashi Prabha Arya and M. K. Singal, More sum theorems for topological

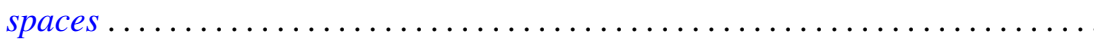

Goro Azumaya, F. Mbuntum and Kalathoor Varadarajan, On M-projective and

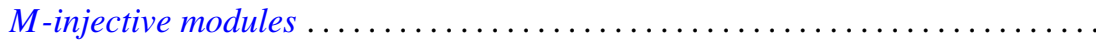

Kong Ming Chong, Spectral inequalities involving the infima and suprema of

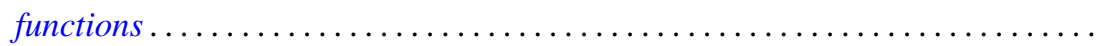

Alan Hetherington Durfee, The characteristic polynomial of the monodromy .......

Emilio Gagliardo and Clifford Alfons Kottman, Fixed points for orientation

preserving homeomorphisms of the plane which interchange two points ......

Raymond F. Gittings, Finite-to-one open maps of generalized metric spaces .......

Andrew M. W. Glass, W. Charles (Wilbur) Holland Jr. and Stephen H. McCleary,

$a^{*}$-closures of completely distributive lattice-ordered groups .............

Matthew Gould, Endomorphism and automorphism structure of direct squares of

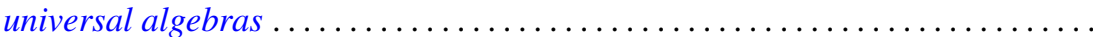

R. E. Harrell and Les Andrew Karlovitz, On tree structures in Banach spaces .....

Julien O. Hennefeld, Finding a maximal subalgebra on which the two Arens

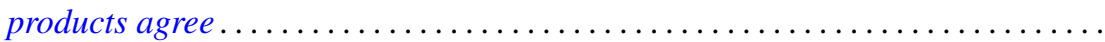

William Francis Keigher, Adjunctions and comonads in differential algebra .......

Robert Bernard Kelman, A Dirichlet-Jordan theorem for dual trigonometric

series

Allan Morton Krall, Stieltjes differential-boundary operators. III. Multivalued operators-linear relations...

Hui-Hsiung Kuo, On Gross differentiation on Banach spaces .

Tom Louton, A theorem on simultaneous observability ...

Kenneth Mandelberg, Amitsur cohomology for certain extensions of rings of algebraic integers.

Coy Lewis May, Automorphisms of compact Klein surfaces with boundary . . .

Peter A. McCoy, Generalized axisymmetric elliptic functions .

211

Muril Lynn Robertson, Concerning Siu's method for solving $y^{\prime}(t)=F(t$, $y(g(t)))$. .

Richard Lewis Roth, On restricting irreducible characters to normal

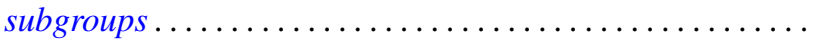

Albert Oscar Shar, $P$-primary decomposition of maps into an $H$-space .

Kenneth Barry Stolarsky, The sum of the distances to certain pointsets on the unit circle.

Bert Alan Taylor, Components of zero sets of analytic functions in $C^{2}$ in the unit ball or polydisc

Michel Valadier, Convex integrands on Souslin locally convex spaces ...

Januario Varela, Fields of automorphisms and derivations of $C$

Arnold Lewis Villone, A class of symmetric differential operators with deficiency

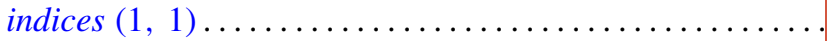

